\title{
DEVELOPMENT OF A SIMULATION APPROACH TO MICROSTRUCTURE EVOLUTION DURING SOLIDIFICATION AND HOMOGENIZATION USING THE PHASE FIELD METHOD
}

\author{
N. Warnken ${ }^{1}$, A. Drevermann ${ }^{2}$, D. $\mathrm{Ma}^{3}$, S. G. Fries ${ }^{4}$, I. Steinbach ${ }^{2}$ \\ ${ }^{1}$ Department of Metallugry and Materials, University of Birmingham, Edgbaston; Birmingham B15 2TT, United Kingdom \\ (formerly ACCESS e.V., Aachen) \\ ${ }^{2}$ ACCESS e.V., Intzestr. 5; 52072 Aachen, Germany \\ ${ }^{3}$ Foundry Institut of the RWTH-Aachen, Intzestr. 5; 52072 Aachen, Germany \\ ${ }^{4}$ SGF Scientific Consultancy; 52072 Aachen, Germany
}

Keywords: Microstructure Modelling, Solidification, Solution Heat Treatment, Phase-Field, CALPHAD, Diffusion, Single

Crystal Solidification

\begin{abstract}
A numerical method for the simulation of microstructure formation during the solidification and solution heat treatment of single crystal superalloys is presented. The method is based on the phase-field method, coupled to thermodynamic calculation (CALPHAD) and kinetic databases. Simulations are performed in a representative transverse section of a directionally solidified dendritic array. Calculated as-cast structures form the bases for the subsequent solution heat treatment simulations. The method is applied to simulate microstructure fromation in a five component model Ni-base superalloy (Ni-5.8Al9.0Cr-7.9Ta-8.8W, wt.\%). Experiments were carried out for validation and good agreement with the model predictions is observed.
\end{abstract}

\section{Overview}

A modelling approach to the simulation of microstructure evolution during solidification and homogenization heat treatment of single crystal superalloys is presented. The approach is based on the phase field method, coupled to CALPHAD type calculations and allows microstructure simulations of complex alloys in two and three dimensions. The application of the model to the processing of a five component model superalloy reveals a good response of the model to changes in the processing conditions. Directional solidification and homogenization heat treatment experiments were carried out in order to validate the simulations. The presented work was part of a larger frame work, aiming at the development of an integrated simulation approach for the manufacture and in service behaviour of coated single crystal superalloys [1].

\section{Modelling approach}

The microstructure evolution of an alloy is mainly determined by three contributions. First the alloy constitution defines the phases, their fractions and compositions; these are properly described by thermodynamics and thus define the energy scale. Second the diffusion of species determines the main contribution of transport of matter and thus provides the time scale. Finally the interface movement determines the morphology and thus sets the length scale of a microstructure. (For simplicity we neglect convection in the melt.) These contributions are modelled using CALPHAD [2, 3], kinetic [4] and phase field modelling [5]. The phase-field implementation in the MICRESS code was used for the simulations presented in this paper $[6,7,5]$.

On the other hand the course of the microstructure evolution is controlled by the process, characterized by the process parameters solidification velocity and temperature gradient during solidifications, and temperature time profiles during the heat treatment. Figure 1 illustrates the modelling approach.

During the directional solidification of superalloys mushy zone lengths of 10th of millimetres are commonly observed. A full coverage of this in $3 \mathrm{D}$, for technically relevant alloys is too challenging, even for todays high performance computers. A more feasible approach is based on a simple analysis of dendritic arrays. These are commonly characterised by the primary dendritic spacing $\lambda_{1}$, which correlates to the solidification parameters, thermal gradient $G$ and solidification velocity $v$ :

$$
\lambda_{1}=c G^{-1 / 2} v^{-1 / 4}
$$

with $c$ being a material dependent coefficient, which is approximatly constant for most Ni-base superalloys [8]. The 


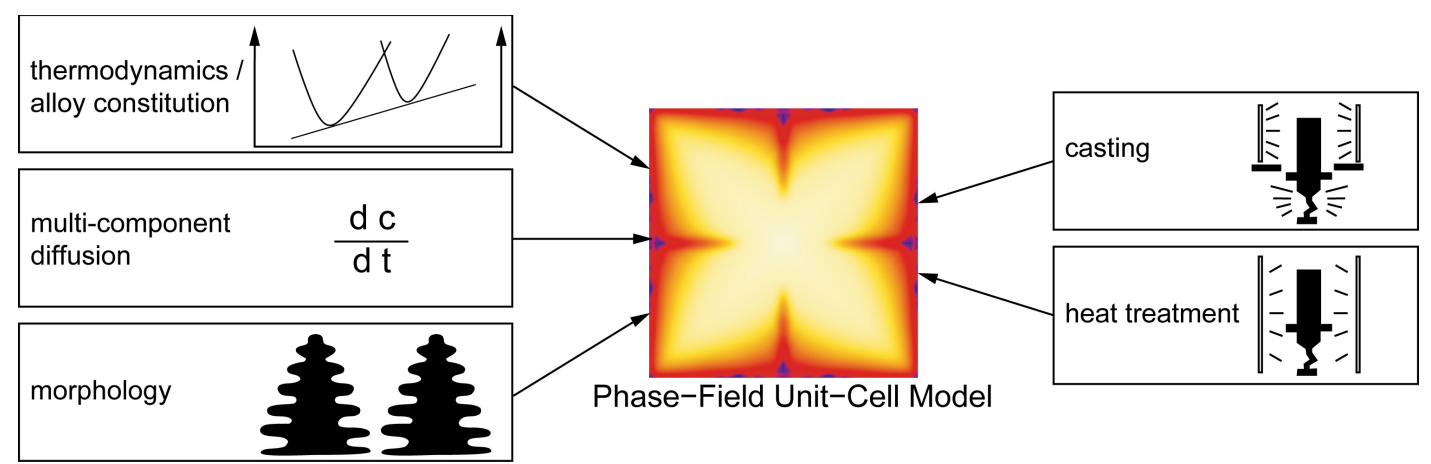

Figure 1: Overview of the modelling approach.

model takes into account the symmetries of directionally growing dendritic arrays; a representative volume element is defined in a transversal (isothermal) section according to the primary dendritic spacing. Due to the dendritic symmetry, only one forth of a dendrite transversal section has to be simulated. Thus the size of the represenative volume element is given by $\left(0.5 \lambda_{1}\right)^{2}$. This is also the distance solute has to travel during the solution heat treatment. This approach was first proposed by Ma et al [9]. The cooling rate is given by $\dot{T}=G v$ and the initial temperature is the dendrite tip temperature, calculated using a extended (multicomponent) marginal stability model $[10,11]$.

Subsequent to solidification, the homogenization heat treatment is simulated. The simulations start from the calculated as-cast microstructure and simulate the equilibration of microsegregation and dissolution of interdendritic $\gamma^{\prime}$ during a non-isothermal temperature time profile. Multicomponent diffusion is taken into account by solving the multicomponent Fick-Onsager equation, whereas the diffusion matrix is calculated from thermodynamic and kinetic databases.

\section{Multiphase-Field Model with CALPHAD Coupling}

An outline of the model used for the simulations presented in this paper is given here. More recent and more detailed description can be found elsewhere $[6,5]$.

Multiphase-Field Model: In phase-field methods the boundary between two phases consists of a diffuse interface described by the phase-field parameter $\phi$. Several phase-field models have been presented in literature which differ in terms of the free energy density in the interfacial region as well as in how they were derived $[12,13,14]$. The following approach is based on a multiphase-field concept which has been published earlier $[15,16]$. The development of the phase-field parameter $\phi_{i}$ of a phase $i$ in a multiphase system is described as the sum of the pair-wise interactions with all other phases $j$ (in the case of a double well potential):

$$
\begin{aligned}
\frac{d \phi_{i}}{d t} & = \\
& \sum_{j}^{N} \mu_{i j}\left[\sigma_{i j}\left(\phi_{i} \nabla^{2} \phi_{j}-\phi_{j} \nabla^{2} \phi_{i}+\frac{36}{\eta_{i j}^{2}} \phi_{i} \phi_{j}\left(\phi_{i}-\phi_{j}\right)\right)\right. \\
& \left.+\frac{6}{\eta_{i j}} \phi_{i} \phi_{j} \Delta G_{i j}\right]
\end{aligned}
$$

Here, $\mu_{i j}$ is the interface mobility, $\sigma_{i j}$ the surface energy, $\eta_{i j}$ the interface thickness, $N$ the number of coexisting phases and $\Delta G_{i j}$ the driving force for the transformation between the phases $i$ and $j$.

A distinctive feature of the multiphase-field model presented here is the coupling to the diffusion equation. The compositions $c_{i}^{k}$ and $c_{j}^{k}$ of species $k$ in phase $i$ and phase $j$ are used to determine the driving force $\Delta G_{i j}$ and the diffusion fluxes instead of the overall composition $c^{k}$. These compositions $c_{i}^{k}$ follow the relation

$$
\sum_{i} \phi_{i} c_{i}^{k}=c^{k}
$$

Multiphase diffusion for a component $k$ is then given as the sum of the fluxes in the individual phases which are calculated from the composition gradients $\nabla c_{i}^{k}$ using only the diagonal terms $D_{i}^{k}$ of the diffusivity matrix:

$$
\frac{d c^{k}(\vec{x}, t)}{d t}=\nabla\left(\sum_{i=1}^{N} \phi_{i} D_{i}^{k} \nabla c_{i}^{k}\right)
$$

CALPHAD Coupling: The thermodynamic calculation needs to provide three inputs from the database,

a) the relation between the phase compositions $c_{i}^{k}$ 
which are in contact,

b) the thermodynamic driving force $\Delta G_{i j}$ for Eq. (2) and

c) conditions for nucleation of secondary phases.

For diffusion-controlled processes a quasi-equilibrium approach is introduced. The driving force $\Delta G_{i j}$ results from a slight deviation from equilibrium. We define the quasiequilibrium by the construction of parallel tangent planes to the Gibbs energy functions $f_{i}$ and $f_{j}$ :

$$
a^{k}=\left(\frac{\partial f_{i}}{\partial c^{k}}\right)_{c_{i}^{k}}=\left(\frac{\partial f_{j}}{\partial c^{k}}\right)_{c_{j}^{k}}
$$

Where $a^{k}$ denotes the slope of the tangent. $\Delta G_{i j}$ is then given by the difference

$$
\Delta G_{i j}=f_{i}-f_{j}+a^{k}\left(c_{i}^{k}-c_{j}^{k}\right)
$$

Equations (3), (5) and (6) are solved to get the quasiequilibrium phase compositions $c_{i}^{k}$ and $c_{j}^{k}$ and the driving force $\Delta G_{i j}$ in a pair-wise manner for all phase interactions $i j$ by use of the software package Themo-Calc [17] which has been enhanced by an extra routine in order to meet also Eq.(3). Together with a realistic database even complex multicomponent and multiphase problems can be treated.

Multi-Binary Extrapolation: The above description provides a complete solution within the local quasi-equilibrium approximation. However, applying that calculation for multicomponent alloys in each time step and for all interface gridpoints implies tremendous calculation times. Therefore, together with the equilibrium calculation the slopes $m_{i j}^{k}$ and $m_{j i}^{k}$ of the liquidus (solvus) and the solidus plane in the directions of every component $\mathrm{k}$ are extracted at the equilibrium compositions $c_{i}^{0, k}$ and $c_{j}^{0, k}$, respectively. By this procedure we get a linearized phase diagram for each interface cell in the calculation domain. These phase diagrams are additionally shifted by the temperature equivalent $\Delta T_{i j}^{\text {off }}$ of the calculated equilibrium deviation $\Delta G_{i j}$ from Eq. (6), where $\Delta S_{i j}^{\text {trans }}$ is the transformation entropy :

$$
\Delta T_{i j}^{\mathrm{off}}=\frac{\Delta G_{i j}}{\Delta S_{i j}^{\mathrm{trans}}}
$$

From the local phase diagrams we get a good extrapolation of the thermodynamic behavior. Time-consuming real equilibrium iterations have to be done only from time to time, when local compositions or the temperature show considerable deviation from the conditions at the last linearisation. From the extrapolation of the equilibrium temperatures from the liquidus plane

$$
T_{i j}^{\mathrm{eq}}=T_{i j}^{0, \mathrm{eq}}+T_{i j}^{\mathrm{off}}+\sum_{k}^{N-1} m_{i j}^{k}\left(c_{i}^{k}-c_{i j}^{0, k}\right)
$$

we obtain the extrapolated driving force $\mathrm{Gij}^{*}$, where $T$ is the actual temperature:

$$
\Delta G_{i j}^{*}=-\Delta S_{i j}^{\mathrm{trans}}\left(T-T_{i j}^{\mathrm{eq}}\right)
$$

Using Eq.(8) and (3), finally the extrapolated local phase compositions can be calculated:

$$
c_{i}^{* k}=\frac{c^{k}-\sum_{j} \phi_{j}\left(c_{j i}^{0, k}-c_{i j}^{0, k} \frac{m_{j i}^{k}}{m_{i j}^{k}}\right)}{\sum_{j} \phi_{j} \frac{m_{j i}^{k}}{m_{j i}^{k}}}
$$

\section{Experiments}

As the paper focuses on the modeling aspects, the validation experiments are only briefly outlined. More detailed results on the experiments will be given in a future publication.

Samples of a five component model superalloy (Table 1) were directionally solidified using a number of different withdrawal speeds $(v=0.03-5.0 \mathrm{~mm} / \mathrm{min})$ and temperature gradients $(G=4-20 \mathrm{~K} / \mathrm{mm})$. The critical $\mathrm{G} / \mathrm{v}$ ratios for

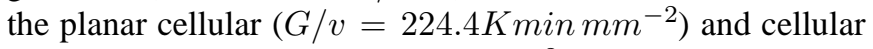
dendritic $\left(G / v=126.3 \mathrm{Kmin}_{\mathrm{mm}}^{-2}\right)$ transitions were determined from quenched samples. In the dendritic regime, microstructures typically consist of $\gamma$ dendrites and interdendritic $\gamma^{\prime}$ (Figure 2 and Figure 3).

\begin{tabular}{cccccc}
\hline & $\mathrm{Al}$ & $\mathrm{Cr}$ & $\mathrm{Ta}$ & $\mathrm{W}$ & $\mathrm{Ni}$ \\
at.\% & 13.06 & 10.49 & 2.66 & 2.92 & bal. \\
wt.\% & 5.80 & 8.98 & 7.94 & 8.84 & bal. \\
\hline
\end{tabular}

Table 1: Composition of the model superalloy in at.\% and wt.\%).

The primary dendritic spacing was measured (Figure 4)and the coefficient in equation 1 was determined as $c=1915$. $10^{-6} m^{3} K^{0.5} s^{-0.25}$. Microsegregation of the alloying elements was characterised by wavelength dispersive X-ray spectroscopy (WDX) elemental mapping. The velocity dependence of the mushy zone length and phase appearance was determined from longitudinal sections of quenched samples (compare figure 2).

Previously directionally solidified samples were homogenised at $1285^{\circ} \mathrm{C}$ and $1295^{\circ} \mathrm{C}$ for up to four hours. Samples were fast cooled to room temperature after different duration and the area fraction of the interdendritic $\gamma^{\prime}$ was measured from micrographs. 


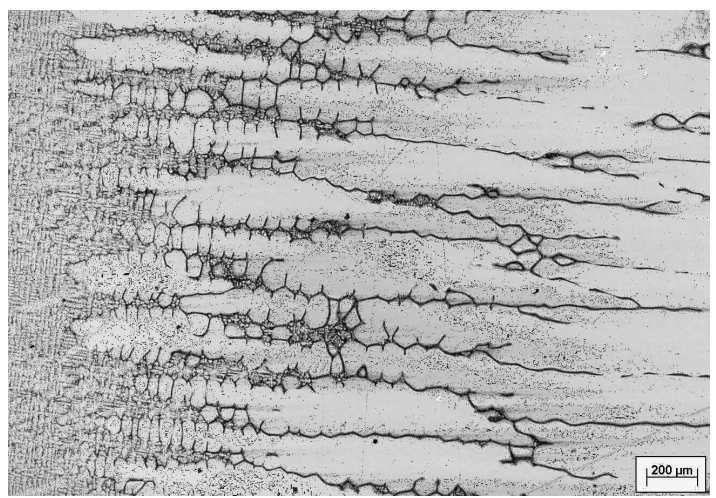

Figure 2: Longitudinal section of directionally solidified and quenched samples $\mathrm{G}=20 \mathrm{~K} / \mathrm{mm}$ and $\mathrm{v}=0.8 \mathrm{~mm} / \mathrm{min}$ ) revealing the solid-liquid interface. The bottom of the mushy zone is outside the image (right hand side).

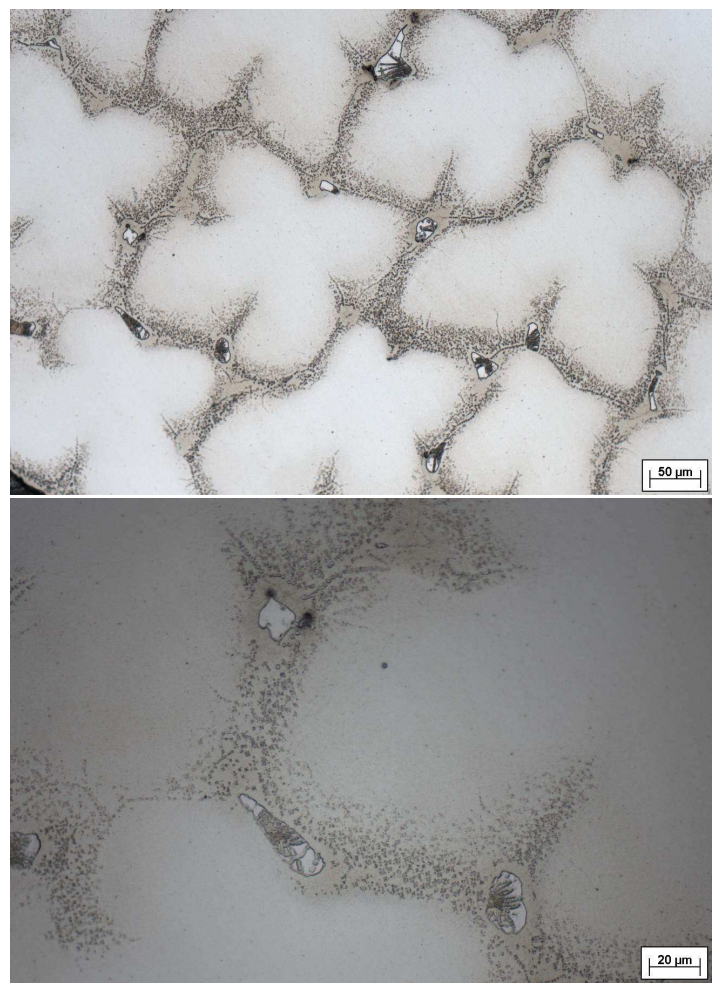

Figure 3: The microstructure of directionally solidified and quenched samples $\mathrm{G}=20 \mathrm{~K} / \mathrm{mm}$ and $\mathrm{v}=0.8 \mathrm{~mm} / \mathrm{min}$ ) reveal $\gamma$ dendrites and interdendritic $\gamma^{\prime}$.

\section{Simulations}

\section{$\underline{\text { Solidification }}$}

Simulations were carried out for the five component model superalloy using different combinations of withdrawal speed and

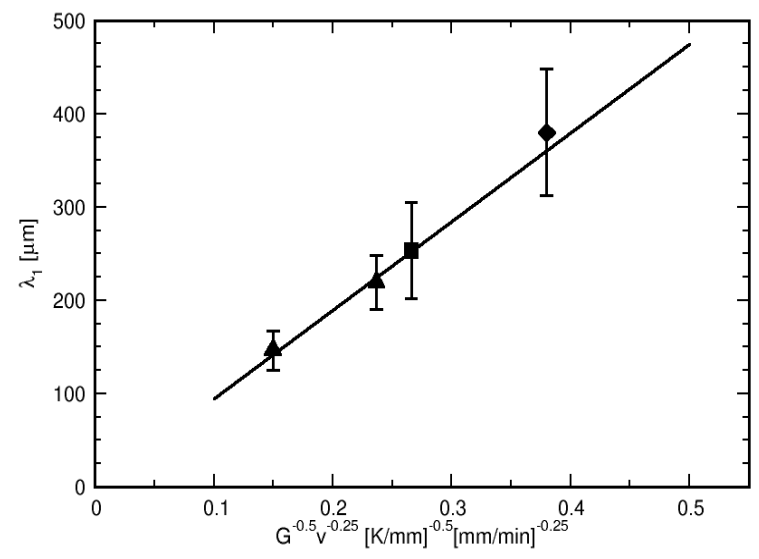

Figure 4: Scaling of the primary dendrite spacing $\lambda_{1}$ with the solidification parameters thermal gradient $G$ and withdrawal rate $v$.

temperature gradients, which were in the same range the experiments. Simulations were done for the alloy composition as given in table 1, and the thermodynamic and kinetic databases published by NIST were used [18, 19].

Figure 5 shows the microstructure evolution during solidification $(G=20 \mathrm{~K} / \mathrm{mm}, v=5.0 \mathrm{~mm} / \mathrm{min})$; $\mathrm{Al}, \mathrm{Cr}$ and Ta enrich in the melt while $\mathrm{W}$ enriches in the dendrite core. In the early stages the initially round dendrite tip breaks up and forms its characteristic fins, which evolve into a typical dendritic cross section. After solidification the microstructure consists of $\gamma$ dendrites and interdendritic $\gamma^{\prime}$. The latter can be identified as yellow spots in the $\mathrm{Al}$ and Ta fields, and blue spots in the $\mathrm{Cr}$ and $\mathrm{W}$ fields. Nucleation of the interdendritic $\gamma^{\prime}$ occurs at the dendrite-liquid interface, as described in [20].

Figure 6 show element composition maps for Ta and W, obtained from a simulation (left hand side) and WDX measurement (right hand side). The color bars represents the same value range for the simulation and the measurement. The interdendritic $\gamma^{\prime}$ can be identified, as described for figure 5. Due to the assumptions made for the representation of the dendritic array the dendritic morphology is less complex in the simulation, but the influence of the dendrite arms on the segregation pattern becomes visible.

A kinetic phase diagram (Figure 7) was obtained from simulations and experiments. It correlates the transition temperatures and the sequence of phase formation during solidification with the solidification parameters. The diagram in Figure 7 was determined for a constant temperature gradient of $G=10 \mathrm{~K} / \mathrm{mm}$. In this graph solid lines without symbols were derived using analytical models, dashed lines are rather speculative, lines with open symbols were obtained from sim- 


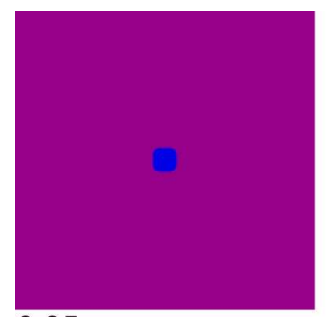

$0.05 \mathrm{~s}$

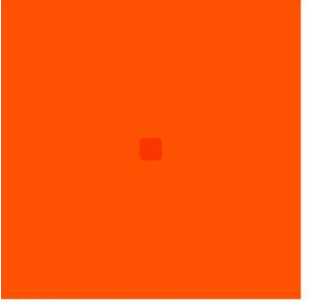

$0.05 \mathrm{~s}$

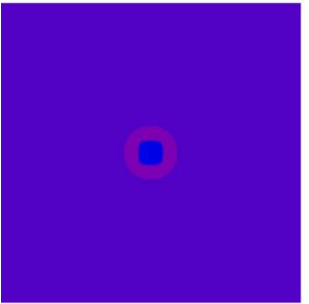

$0.05 \mathrm{~s}$

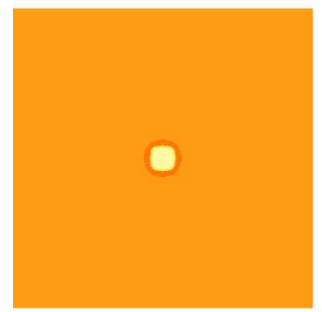

$0.05 \mathrm{~s}$

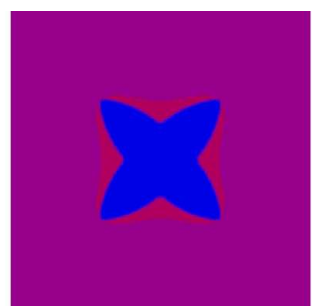

$0.50 \mathrm{~s}$

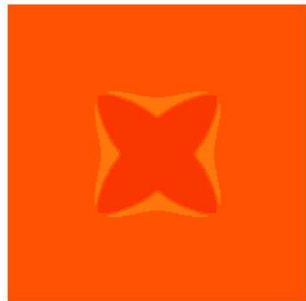

$0.50 \mathrm{~s}$

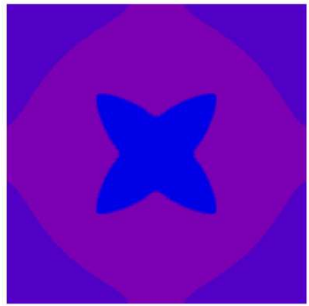

$0.50 \mathrm{~s}$

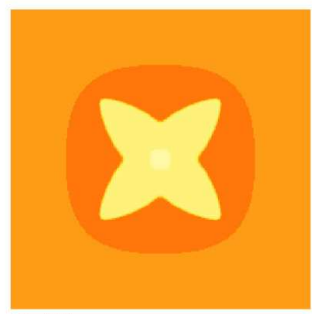

$0.50 \mathrm{~s}$

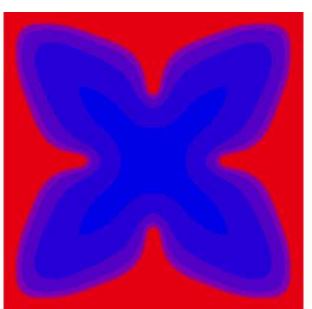

$15.00 \mathrm{~s}$

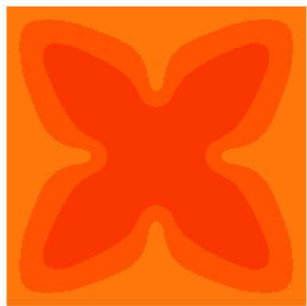

$15.00 \mathrm{~s}$

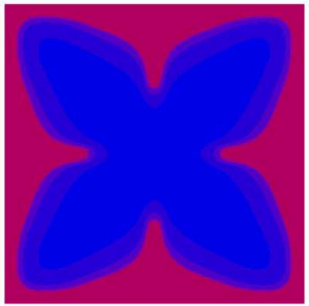

$15.00 \mathrm{~s}$

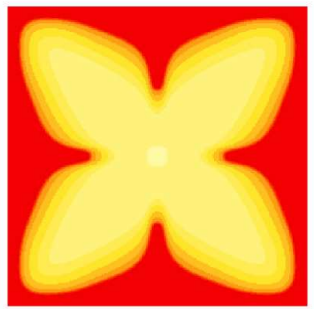

$15.00 \mathrm{~s}$
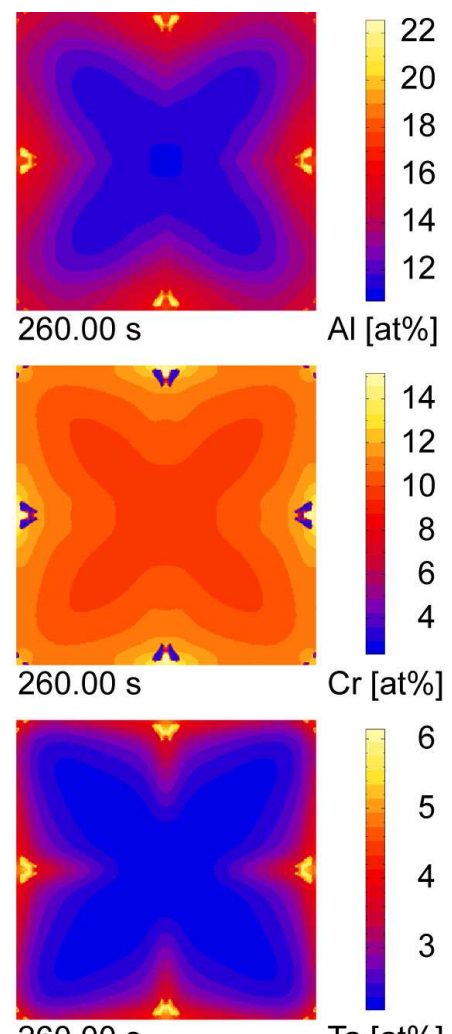

$260.00 \mathrm{~s}$

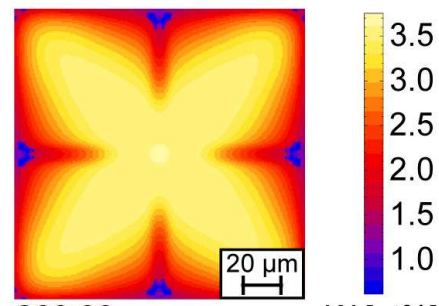

260.00 s

W [at\%]

Figure 5: Evolution of the morphology and microsegregation during solidification; the colors denote the atomic fractions of Al, $\mathrm{Cr}$, Ta and W, respectively. The yellow (Al, Ta), respectively blue $(\mathrm{Cr}, \mathrm{W})$ spots indicate the location of the interdendritic $\gamma^{\prime}$.

ulations and with lines with closed symbols from experiments. In the planar solidification front regime $(v<0.045 \mathrm{~mm} / \mathrm{min})$ the transition temperatures are given by the equilibrium phase diagram; the solid-liquid interface moves at the solidus temperature [21] and the crystal is microsegregation free. Thus $\gamma^{\prime}$ forms only in a solid state reaction at the $\gamma^{\prime}$ solvus temperature and below. An increase of the solidification velocity above the planar front stability limit, leads to the formation of cellular growth morphologies, the solidified crystal exhibits microsegregation. Above $v>0.08 \mathrm{~mm} / \mathrm{min}$ the solidification front exhibits dendritic morphologies. The dendrite tip temperature defines the liquidus line in the diagram. The solidifi- cation interval increases with the solidification velocity. This corresponds to an increase of the mushy zone length, which were experimentally determined from longitudinal sections of quenched samples (figure 7 closed square symbols). From these also the temperature of $\gamma^{\prime}$ formation was determined (figure 7 circlular symbols). The formation of interdendritic $\gamma^{\prime}$ is only observed above a threshold velocity of $v=0.3 \mathrm{~mm} / \mathrm{min}$ and $\gamma^{\prime}$ forms at a temperature approximatly $10 K$ higher than predicted by Scheil. The $\gamma^{\prime}$ formation temperature is independent from the solidification rate, in both experiments and simulations. This effect occurs due to backdiffusion in the solid. The kinetic phase diagrams obtained from experiments 

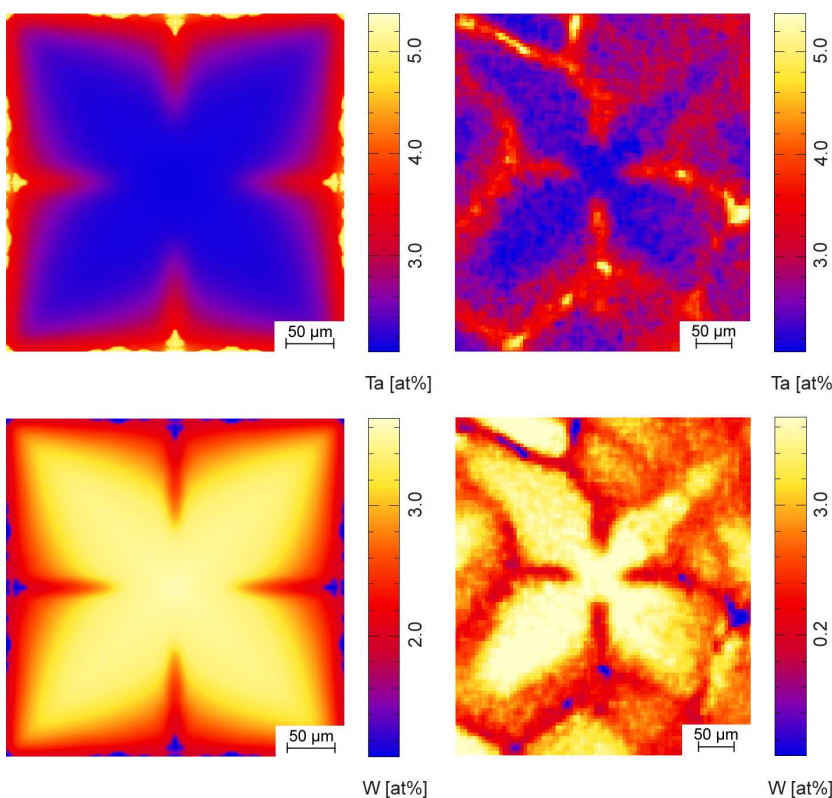

Ta [at\%]

$W[a t \%]$

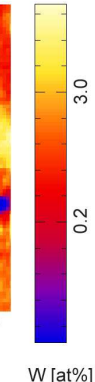

Figure 6: Comparison of composition maps for Ta and W, calculated (left hand side) and measured by WDX (right hand side). Solidification paramters were $G=4.0 \mathrm{~K} / \mathrm{mm}$ and $v=3.0 \mathrm{~mm} / \mathrm{min}$

and simulations are in good agreement, especially above the $\gamma^{\prime}$ formation threshold. Less good agreement below this point is due to the fact that the growth morphology is rather cellulardendritic than fully dendritic, but the model was developed for dendritic structures.

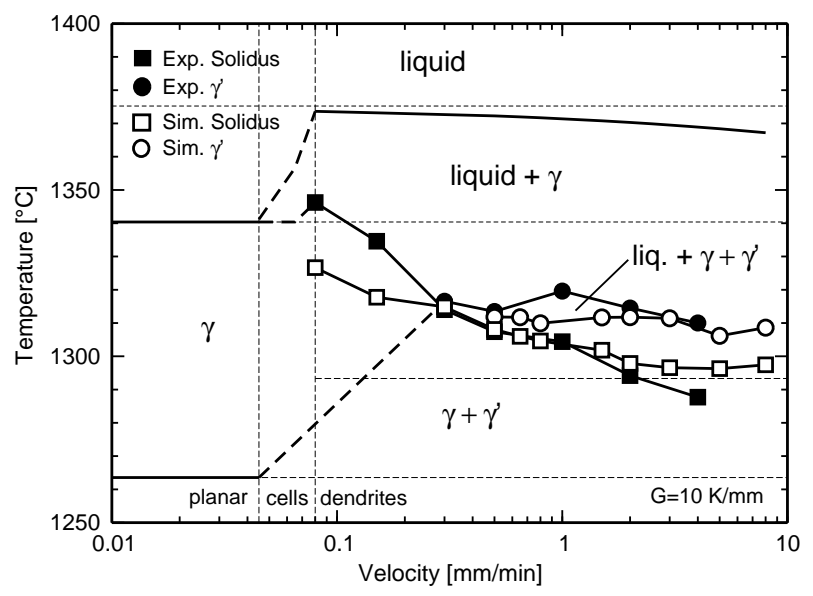

Figure 7: The kinetic phase diagram as obtained from simulations and experiments.
The presented model provides a tool to investigate microstructure formation during solidification with modest efford. As the solidification parameters thermal gradient $G$ and solidification velocity $v$ are transformed into the model parameters primary spacing $\lambda_{1}$, cooling rate $\dot{T}$ and initial temperature, the response of the model to changes in the solidification parameters is not quite obvious.

Figure 8 show results of a systematic variation of the solidification parameters, respectively the model parameters and their effect on the $\gamma^{\prime}$ fraction. Each symbol in the graphs stands for one simulation. The $\gamma^{\prime}$ fraction is plotted over the primary spacing. It is found that the $\gamma^{\prime}$ fraction is not a unique function of the primary spacing. Different combinations of $G$ and $v$ yield similar primary spacings, but different $\gamma^{\prime}$ fraction. Changes in the process parameters are not entirely compensated by changes in the primary spacing, as one might initially expect.

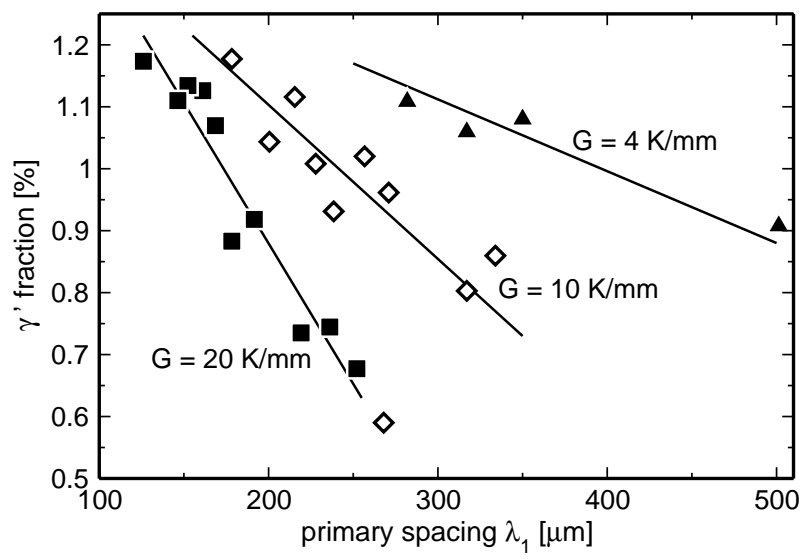

Figure 8: Phase fraction of $\gamma^{\prime}$ as functions of the dendrite spacing for different thermal gradients and solidification velocities.

The the strength of the phase-field approach is, that simulations are not restricted to $2 \mathrm{D}$. A simple $3 \mathrm{D}$ the unit-cell can be derived by giving the $2 \mathrm{D}$ representative volume element a thickness, which itself is given by the secondary dendrite arm spacing $\lambda_{2}$. In this case the interdendritic $\gamma^{\prime}$ forms between the secondary dendrite arms (Figure 9). The interdendritic $\gamma^{\prime}$ fraction is slightly lower than in the 2D case, due to enhanced backdiffusion. However, the $3 \mathrm{D}$ extention increases the CPU time of the simulations by approximatly a factor of 30 .

For larger calculation domains more than one dendrite can be modelled (figure 10). The primary spacing condition is fullfilled if the number of dendrites is adjusted to the size of the calculation domain. The condition to be fullfilled is the:

$$
\lambda_{1}=\sqrt{\frac{A}{n}}
$$




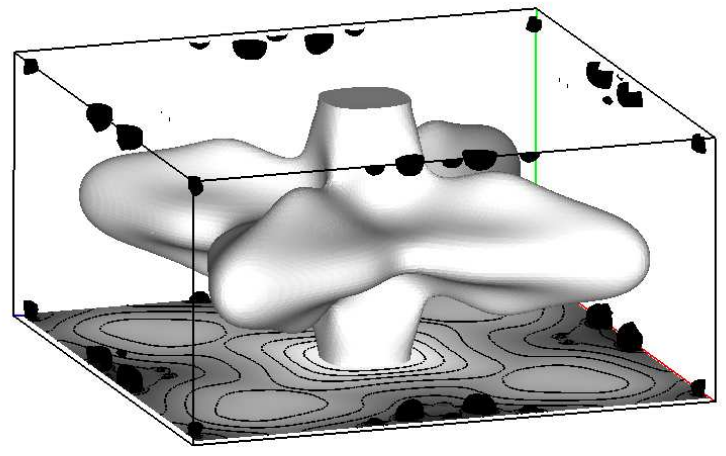

Figure 9: Result of a 3D simulation illustrating the dendrite morphology, location of interdendritic $\gamma^{\prime}$ and $\mathrm{W}$ mapping at the bottom of the calculation domain. The morphology is revealed by plotting an appropriate $\mathrm{W}$ concentration isosurface.

with $A$ being the area of the calculation domain and $n$ the number of dendrites. The other conditions, cooling rate and initial temperature, remain the same as outlines in the modelling section. If dendrites with different orientation are modelled, the formation of a grain boundary can be simulated (figure 10).

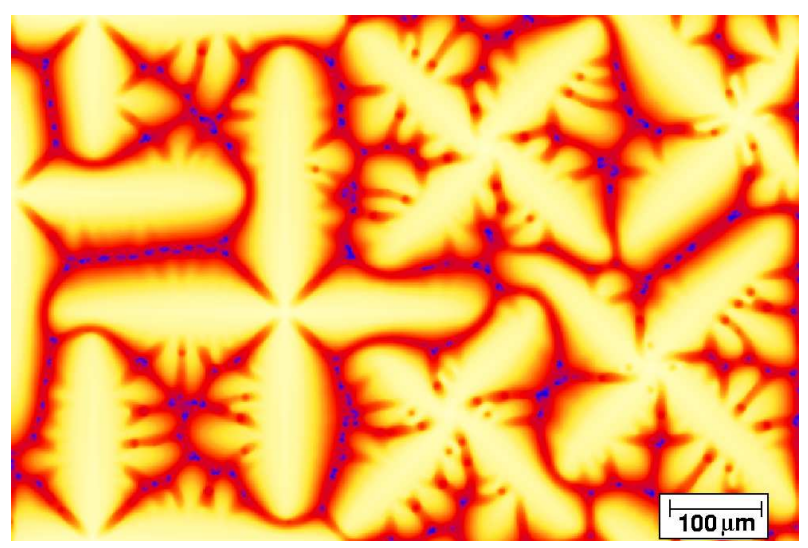

Figure 10: Simulation result obtained for a larger simulation domain. Two grains with different orientations form a grain boundary. The $\mathrm{W}$ composition map is shown, blue spots indicate interdendritic $\gamma^{\prime}(G=20 \mathrm{~K} / \mathrm{mm}, v=5.0 \mathrm{~mm} / \mathrm{min})$.

\section{$\underline{\text { Solution Heat Treatment }}$}

Starting from the calculated as-cast structure the solution heat treatments are simulated. An initial fast ramp is followed by a simple isothermal holding at $1285^{\circ} \mathrm{C}$ (figure 11). The ascast structure was taken from the simulation shown in figure 5 . The elements $\mathrm{Al}$ and $\mathrm{Cr}$ diffuse almost an order of magnitude faster than $\mathrm{Ta}$ and $\mathrm{W}$. After the ramp $\mathrm{Al}$ and $\mathrm{Cr}$ are almost homogeneous (30 min.). Depleted spots appear in the $\mathrm{Al}$ and $\mathrm{Cr}$ fields ( 1 hour), which shift their location towards the dendrite core ( 2 hours). This effect is due to the cross diffusion effect, the gradients in the $\mathrm{W}$ field drive the diffusion of $\mathrm{Al}$ and $\mathrm{Cr}$. Thus the the overall homogenization is controlled by W diffusion, after a certain point the other elements only follow. After 10 hours of heat treatment the degree of microsegregation is significantly reduced, but some interdendritic $\gamma^{\prime}$ remains.

Isothermal solution heat treatments were simulated for $1275^{\circ} \mathrm{C}, 1285^{\circ} \mathrm{C}, 1295^{\circ} \mathrm{C}$ and $1305^{\circ} \mathrm{C}$. Figure 12 show the calculated $\gamma^{\prime}$ fraction as functions of time (lines). The $\gamma^{\prime}$ fractions obtained from experiments conducted at $1285^{\circ} \mathrm{C}$ and $1295^{\circ} \mathrm{C}$ are given as symbols. The error bars indicate the standard deviation of the measurement. At first glace the error bars appear hugh, but it should be noted that the overall $\gamma^{\prime}$ fraction is rather low (around 1.5\%).

The simulation results show an increase of the $\gamma^{\prime}$ fraction at the early stages of the simulation $\left(1275^{\circ} \mathrm{C}\right.$ and $\left.1285^{\circ} \mathrm{C}\right)$. As $\gamma^{\prime}$ can only form during solidification, the $\gamma$ matrix can not decompose into a $\gamma / \gamma^{\prime}$ mixture (secondary $\gamma^{\prime}$ formation). Thus at sufficiently low temperatures the interdendritic $\gamma^{\prime}$ growths instead, although slowly. However, this indicates that at the given temperatures $\left(1275^{\circ} \mathrm{C}\right.$ and $\left.1285^{\circ} \mathrm{C}\right)$ the interdendritic $\gamma^{\prime}$ should be surrounded by a $\gamma / \gamma^{\prime}$ mixture, which itself is stabilised by the presence microsegregation. After sufficiently long time microsegregation get homogenised by diffusion and the interdendritic $\gamma^{\prime}$ starts to dissolve. This can be regarded as an incubation time for the $\gamma^{\prime}$ dissolution. The experiment at $1285^{\circ} \mathrm{C}$ shows the same incubation time. Increasing temperatures shorten in the incubation time, until it become absent $\left(\left(1295^{\circ} \mathrm{C}\right.\right.$ and $\left.\left.1305^{\circ} \mathrm{C}\right)\right)$. For these temperatures the $\gamma^{\prime}$ fraction decreases right from the start of the hold. Again the experiment shows similar behaviour.

\section{Summary and Discusion}

A model for the simulation of microstructure evolution during directional solidification and homogenization heat treatment is proposed and applied to a five component model superalloy. The model is based on a representative volume element (2D) for an directionally solidified dendritic array.

As-cast structures are calculated and compared to experimental ones. Generally good agreement is found. Despite 

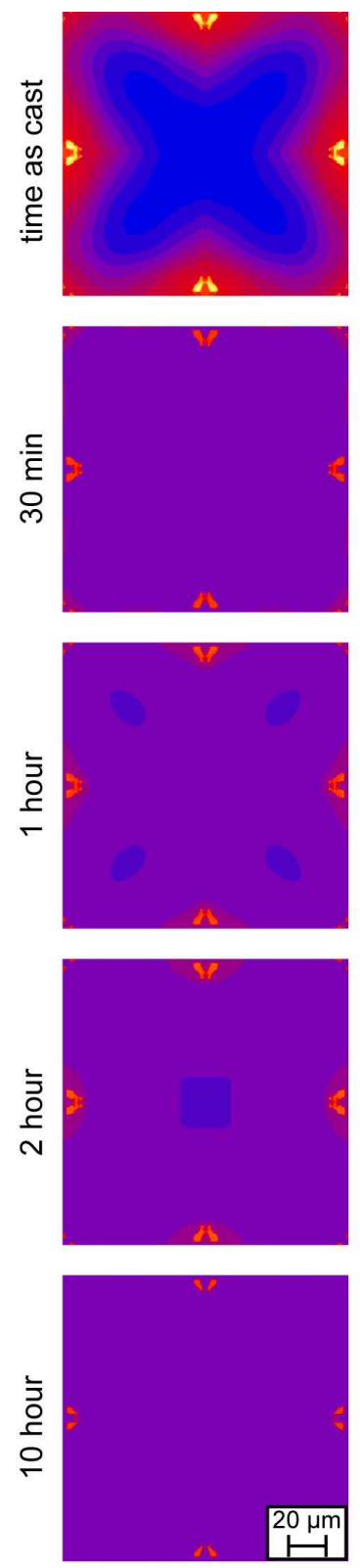

121416182022

Al [at\%]
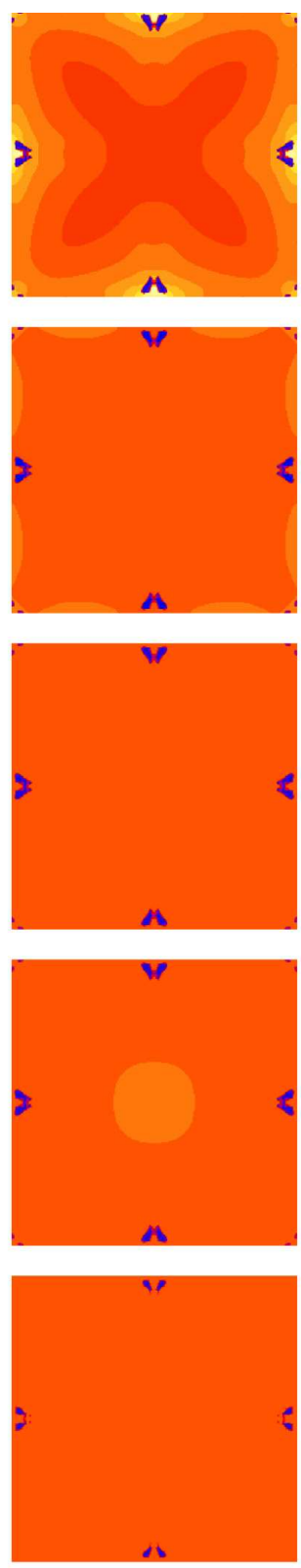

$\begin{array}{llll}4 & 6 & 8 & 1012\end{array}$
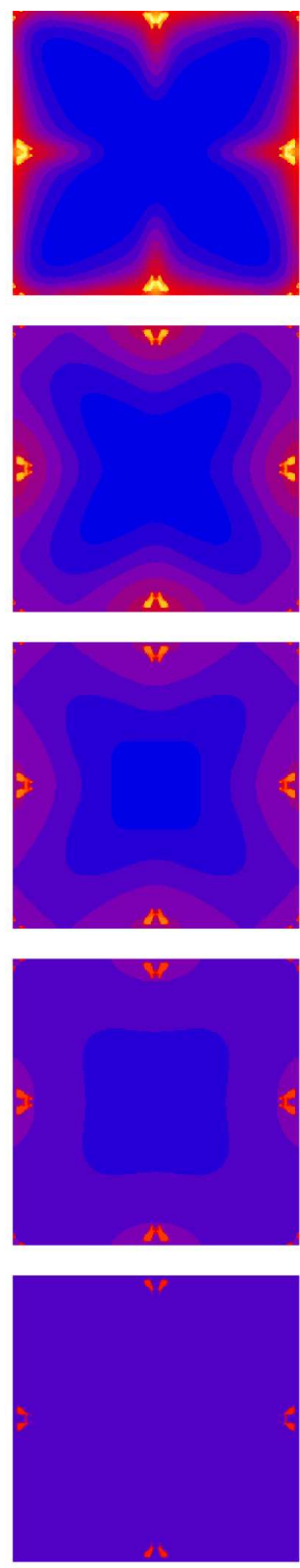

$$
\begin{array}{llll}
3.0 & 4.0 & 5.0 & 6.0
\end{array}
$$
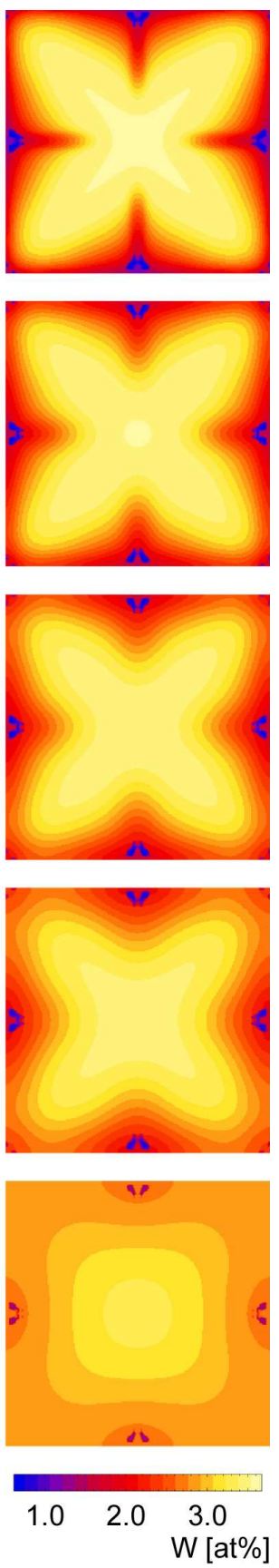

Figure 11: Evolution of the spatial element distribution during the solution heat treatment, isothermal holding at $1285^{\circ} \mathrm{C}$. The as-cast microstructure is the same as in figure 5 .

its simplicty the model reproduces the complex interactions which lead to the solidification velocity dependent mushy zone length and sequence of phase formation. It is demonstrated that the method can be easily extended to 3D.
The calculated as-cast structure is used as the starting condition for the homogenization heat treatment simulation. Experimentally observed behaviour is well reproduced by the simulations. The $\gamma^{\prime}$ dissolution kinetics during solution heat treat- 


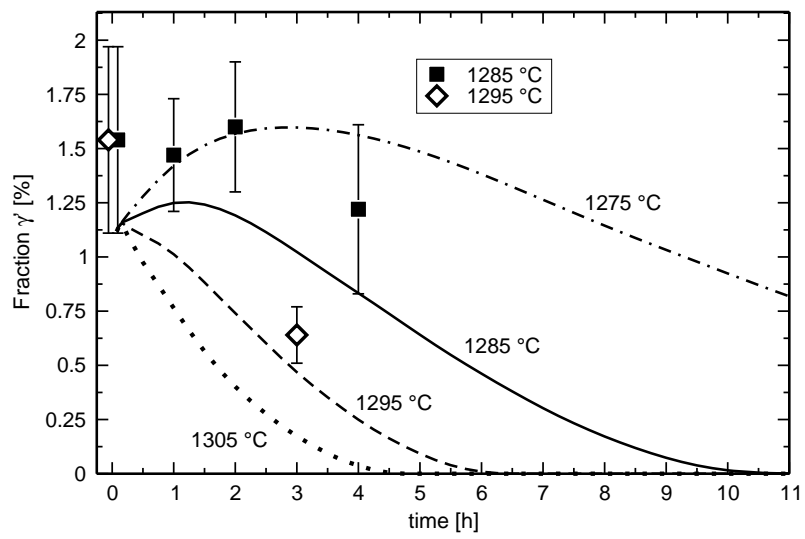

Figure 12: Comparison of interdendritic $\gamma^{\prime}$ fraction as a function of time, as obtained from simulations (lines) and experiments (symbols with error bars), for various temperatures.

ment show a temperature dependent incubation period, due to the presence of microsegregation which has to be homogenised before the interdendritic $\gamma^{\prime}$ starts to dissolve. This behaviour is in agreement with experimental observations.

Although a 2D representation is used for the 3D structure of a dendritic array, the simulations provide useful results. The next challenge in the model development is the application of the method to commercial superalloys and the validation against technical production processes.

\section{References}

[1] R. Herzog, N. Warnken, I. Steinbach, B. Hallstedt, C. Walter, J. Muller, D. Hajas, E. Munstermann, J. M. Schneider, R. Nickel, D. Parkot, K. Bobzin, E. Lugscheider, P. Bednarz, O. Trunova, and L. Singheiser. Integrated approach for the development of advanced, coated gas turbine blades. Adv. Eng. Mater., 8(6):535-562, June 2006.

[2] U. R. Kattner. The thermodynamic modeling of multicomponent phase equilibria. JOM, 49(12):14-19, December 1997.

[3] H.L. Lukas, S.G. Fries, and B. Sundman. Computational Thermodynamics. Cambridge University Press, 2007.

[4] Jan-Olof Andersson and John Agren. Models for numerical treatment of multicomponent diffusion in simple phases. J. Appl. Phys., 72(4):1350-1355, 1992.

[5] I. Steinbach, B. Böttger, J. Eiken, N. Warnken, and S. G. Fries. Calphad and phasefield modeling: A success- ful liaison. Journal of Phase Equilibria and Diffusion, 28(1):101-106, 2007.

[6] J. Eiken, B. Bottger, and I. Steinbach. Multiphasefield approach for multicomponent alloys with extrapolation scheme for numerical application. Phys. Rev. E, 73(6):066122-9, June 2006.

[7] MICRESS. Microstructure evolution simulation software: Micress. web-page: www.micress.de, 2004.

[8] D. Goldschmidt. Single-crystal blades for gas-turbines .1. casting process and microstructure. Materialwiss. Werkstofftech., 25(8):311-320, August 1994.

[9] D. Ma. Modellierung des dendritenwachstums und der mikroseigerung bei gerichteter erstarrung, teil i: Entwicklung eines analytischen mikromodells. Gießereiforschung, 1:29-34, 1998.

[10] M. Bobadilla, J. Lacaze, and G. Lesoult. Influence des conditions de solidification sur le deroulement de la solidification des aciers inoxydables austenitiques. J. Cryst. Growth, 89(4):531-544, July 1988.

[11] H. Diepers, J. Eiken, and I. Steinbach. The effect of thermodynamics and kinetics on the dendritic structure in ternary fe-c-mn. In M. R. Jolly D. M. Stefanescu, J. A. Warren and M. J. Krane, editors, Modeling of Casting, Welding and Advanced Solidification Processes- $X$, pages 29-36, 2003.

[12] A. A. Wheeler, W. J. Boettinger, and G. B. McFadden. Phase-field model for isothermal phase transitions in binary alloys. Phys. Rev. A, 45(10):7424-, May 1992.

[13] G. Caginalp and W. Xie. Phase-field and sharp-interface alloy models. Phys. Rev. E, 48(3):1897-1909, September 1993.

[14] Seong Gyoon Kim, Won Tae Kim, and Toshio Suzuki. Phase-field model for binary alloys. Phys. Rev. E, 60(6):7186-7197, December 1999.

[15] I. Steinbach, F. Pezzolla, B. Nestler, M. See[ss]elberg, R. Prieler, G. J. Schmitz, and J. L. L. Rezende. A phase field concept for multiphase systems. Physica D: Nonlinear Phenomena, 94(3):135-147, July 1996.

[16] J. Tiaden, B. Nestler, H. J. Diepers, and I. Steinbach. The multiphase-field model with an integrated concept for modelling solute diffusion. Physica D: Nonlinear Phenomena, 115(1-2):73-86, April 1998. 
[17] B. Sundman, B. Jansson, and J. O. Anderson. The thermo-calc databank system. CALPHAD, 9:153-190, 1985.

[18] U.R. Kattner. Construction of a thermodynamic database for ni-base superalloys: a case study. In R.D. Shull P.E.A. Turchi, A. Gonis, editor, CALPHAD and Alloy Thermodynamics, pages 147-164. TMS Warrendale (PA), 2002.

[19] C. E. Campbell, W. J. Boettinger, and U. R. Kattner. Development of a diffusion mobility database for ni-base superalloys. Acta Mater., 50(4):775-792, February 2002.

[20] N. Warnken, D. Ma, M. Mathes, and I. Steinbach. Investigation of eutectic island formation in sx superalloys. Materials Science and Engineering: A, 413-414:267271, December 2005.

[21] W. Kurz and D. J. Fisher. Fundamentals of Solidification. Trans Tech Publications, 1998. 